\title{
Sleep Support System for Providing Comfortable Sleep Environment Using a Single-Channel EEG Sensor
}

\author{
Kohei Fujimoto ${ }^{\mathrm{a}}$, Keiji Matsumoto ${ }^{\mathrm{a}^{*}}$

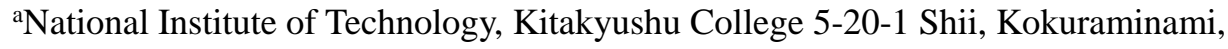 \\ Kitakyushu, Fukuoka, 802-0985, Japan \\ *Corresponding Author:kmatsu@kct.ac.jp
}

\begin{abstract}
In this study, we develop a sleep monitoring system that controls indoor equipment to maintain comfortable sleeping conditions during the sleep-state transition estimation. Although bio-information (such as body-motion and heartbeat) is conventionally needed to estimate sleep transitions, in this study we rely solely upon a simple singlechannel EEG sensor. The Index of Brain Resting State (IBRS), calculated from the subject's EEG frequency spectrum, is used for estimating the subject's state. The developed Sleep Support System employs LED lighting installed in a darkroom as an output instrument that automatically turns off when the subjects move from the arousal state to sleep onset.
\end{abstract}

Keywords: sleep stage, EEG, control, IBRS.

\section{Introduction}

Sleep is a basic biological phenomenon, which plays a vital role in the recovery of the mind and body from fatigue. Despite this fact, recent surveys conducted by the Japan Preventive Association of Life-style related Disease reveals that the average sleep time of people has been decreasing, and one person in about five has sleep disorders to some extent [1]. It is known that lack of sleep can reduce concentration power and can trigger many types of diseases as the result of a weakened immune system [2]. In the case of infants, low quality sleep has a bad influence on the growth of the body and brain. An effective solution needs to be found to counter this problem, because the number of people with sleep disorders seems to be increasing with time. Generally, sleep is monitored using the polysomnography
(PSG) method [3]. This method is based on the analysis of brainwaves, eye movements, and the electromyogram, and it determines sleep-depth by simultaneously measuring many types of bio-information, such as heartbeat, aspiration, and body motion. However, the physical examination process at specialized institutions using the PSG method can be strenuous for the patients, because it requires them to wear many electrodes. Some improved methods have been devised for easy measurement of the sleep. In order to estimate the sleep onset, the research with respect to the sleep detection using parameters obtained from the acoustic signals such as intracorporeal sound have been conducted [4,5]. In other research purposing extraction of novel sleepstage information, the relationship between the autonomous nerve rhythm and brainwave in terms of the frequency analysis of $20 \mathrm{~Hz}$ or more using modify-wavelet-transform have been reported. All of these methods focus on evaluating the sleep-state.

In this study, we develop a system that controls the equipment to maintain comfortable sleep during the sleepstate transition estimation. As mentioned above, although bio-information, such as body-motion and heartbeat, is needed to estimate the sleep, we only use the simple singlechannel EEG sensor. We propose the Sleep Support System, because this system has a possibility to help the people having sleep trouble as well as healthy people who desire to maintain their present condition.

\section{Method}

\subsection{EEG device}

Traditional EEG experiments are usually impractical in real-world settings because they often use multi-channel 
electrodes for accurate measurement. For this reason, it seems difficult to realize the applied product of EEG feature using it easily. In this experiment, a single-channel dry EEG sensor manufactured by NeuroSky Inc. placed on the forehead is used as a user-friendly interface. The singlechannel EEG sensor has two electrodes to measure brain potentials; an active electrode and a reference electrode. The EEG sensor is worn on the head of the test subject, and it monitors the electrical potential between the electrodes attached on the forehead (active) and the earlobe (reference). It is possible to analyze each frequency elements in detail, because the EEG signals are presented at every $0.25 \mathrm{~Hz}$ in the range of 0 to 255 frequency band [7].

\section{$2.2 \quad$ Sleep EEG}

Generally, the brainwaves during sleep, which are different from that of the arousal state, have several characteristics depending on the stage of the sleep depth [8,9]. The closed-eye wakefulness before sleep mainly generates the alpha wave. When the human becomes increasingly drowsy, the arousal-state's alpha wave decreases gradually. Instead, a low-level theta wave and beta wave are observed. This is called the first stage of sleep. When you begin falling deeper into sleep, a slow wave of high-level voltage called Vertex Sharp Transient is generated gradually. In the second stage of sleep, a brainwave from 12 to $14 \mathrm{~Hz}$ called the spindle wave is observed fitfully for a period of a few seconds and the Vertex Sharp Transient disappears fully and the spindle wave becomes stable in this stage. When the sleep advances to the next stage, a brainwave of around four $\mathrm{Hz}$, which is called Sharp Transient of Sleep (POSTS), rhythmically appears. In this study, we assume that the period between the closed-eye wakefulness and second-stage of sleep is the hypnagogic. We expect that the brainwave's frequency elements change as following. In the stage of closed-eye wakefulness, the alpha wave is dominant compared to other frequency elements. The theta wave and beta wave are generated occasionally in the next stage of sleep, and by the second-stage of sleep, the amplitude of the theta wave becomes higher than that of the alpha wave. These continuous movements of the frequency components are measured to estimate the hypnagogic.

\subsection{IBRS}

The IBRS, which is the electroencephalographic index to evaluate the resting state of the human, was proposed by Nakamura et al [10]. Figure.1 shows the conceptual diagram of the human state and brainwave frequencies. Generally, the alpha band is electroencephalographically used as an index of rest, using its quantity of magnitude as a discrimination criterion. However, further brainwave information is required for correct estimation of the human state because the alpha band is considerably affected by visual information [11]. Generally, the beta band components increases in amplitudes and its frequencies change into fast wave oscillations if the brain enters an aroused and excited state. Conversely, when the brain becomes drowsy and enters a suppressed state, the theta band components increases in amplitudes and its frequencies change into slow wave oscillations. The condition between arousal and drowsiness is thought as the brain's resting state. Therefore, the magnitude of the alpha band compared to the theta and beta bands is defined as the IBRS. The mathematical definition of the IBRS is given in equations (1) and (2).

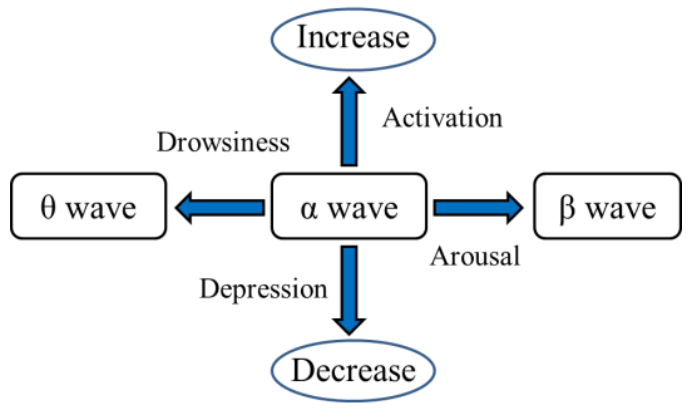

Fig.1 Relationship between the human state and brainwave frequencies

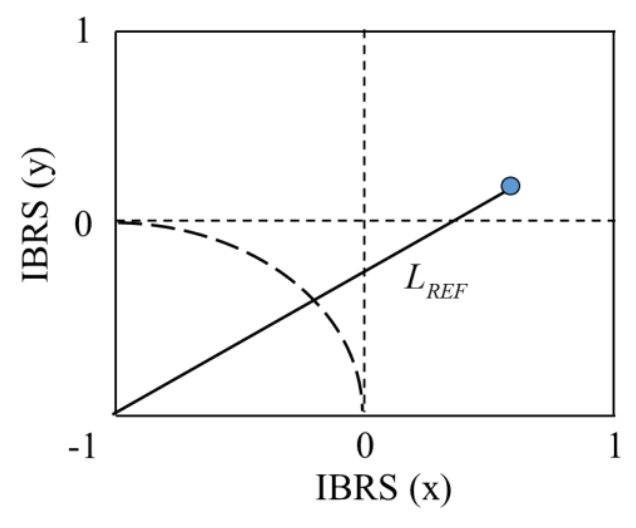

Fig.2 2D display of the IBRS.

$$
\begin{gathered}
X_{I B R S}=\frac{\theta_{s t r}-\alpha_{s t r}}{\theta_{s t r}+\alpha_{s t r}} \\
Y_{I B R S}=\frac{\beta_{s t r}-\alpha_{s t r}}{\beta_{s t r}+\alpha_{s t r}}
\end{gathered}
$$


The IBRS is visualized in two dimensions using the ratio between the theta wave and the alpha wave or between the beta wave and the alpha wave. The horizontal axis is defined as the IBRS's $x$ component, represented by both the theta wave and alpha wave, while the vertical axis is defined as the IBRS's y component, represented by both the beta wave and the alpha wave. Both the IBRS's components are represented in a range from -1 to +1 . The value gradually approaches -1 as the alpha wave increases, taking the point $(-1,-1)$ as the origin. The IBRS increases when the $(x, y)$ coordinate becomes close to this origin. The vector length from the origin is defined as the IBRS, as shown in Fig.2. We estimate the human state by using this index. Although the IBRS is normally used for estimating the brainwave under arousal state, the value is employed as an index of sleep onsets in this experiment [11]. As described above, the closed-eye wakefulness before sleep mainly generates the alpha wave. When the human becomes gradually drowsy, it is thought that the theta wave relatively increases more than alphawave. Therefore, it is possible to detect the sleep onsets using the IBRS.

\subsection{Designed Sleep Support System}

Fig. 3 and Fig. 4 show a configuration diagram of the designed system and the appearance of the fabricated system, respectively. The Sleep Support System consists of an EEG sensor, a Bluetooth module, a microcomputer, a dark-room, an LED drive circuit, and a high-luminance LED. An Arduino is employed as the microcomputer. The dark-room constructed for this experiment was $2.0 \mathrm{~m}$ long $\times 1.2 \mathrm{~m}$ wide $\times 2.1 \mathrm{~m}$ high. A cot bed was installed in the dark-room so that the subject could relax naturally and sleep comfortably. The LED drive circuit was designed to control the brightness of the LED fixture (EdiPowerII, 16-24W, EPSW-VF55) that was installed in the ceiling of the dark-room, providing light of brightness up to $5301 \mathrm{x}$. The chain of signal processing begins with the detection of the subject's brainwave using the EEG sensor. The data obtained by the EEG is transmitted to the Arduino via Bluetooth. The Arduino computes the IBRS using the EEG data by analyzing the three frequency components of the brain waves - the alpha wave, beta wave, and theta wave - to estimate the subject's sleep state. The signal estimating the subject's sleep state is then transmitted from the Arduino to the LED drive circuit. In accordance with this signal, the LED drive circuit either turns on the LED (in the case of the arousal state being detected) or turns it off (in the case of the sleep state being detected). The experimental procedure consisted of the subject, wearing the
EEG sensor, entering the dark-room, lying down on the bed, and closing his eyes, while the proposed sleep support system monitored his sleep state. The monitoring period selected for the experiment was approximately $10 \mathrm{~min}$ as the estimation of the initial transition from the arousal state to the sleep state using solely the EEG was the main focus of this research.

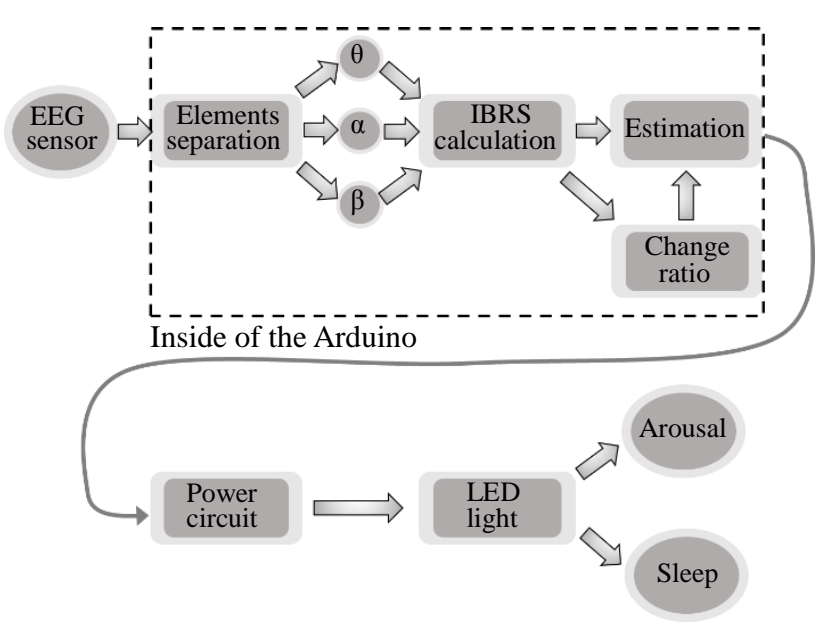

Fig.3 Configuration of designed Sleep Support System

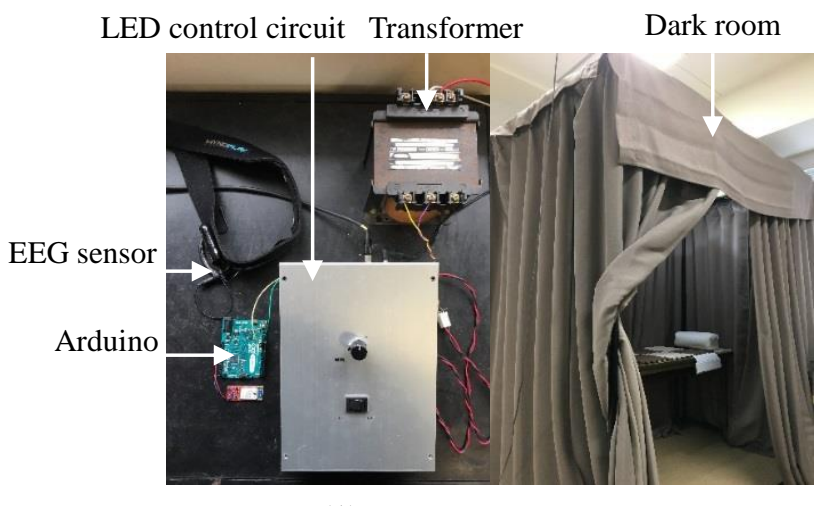

(1)

(2)

Fig.4. Fabricated Sleep Support System (1) control circuit (2) dark room

\section{Result and discussion}

Figure. 5 shows the transition of the magnitude of the three types of EEG in the time domain. Every frequency element looks a little complicated because brainwaves are always fluctuating. To understand the relationship between the human state of sleep and a human's EEG, observation from a broad perspective is important. From this point of view, the amplitude of the average values per minute is plotted in Fig.6. It seems that the average value of the alpha wave until the first 270 seconds in Fig.6 is higher than 5.3. By contrast, the average values of the theta wave and beta 
wave in the same period are respectively lower than 2.9 and 0.55. It is thought that the subjects were in the stage of closed-eye wakefulness, because the alpha wave was higher than the theta wave and beta wave in this period. However, from 270 second onwards, the amplitude of the alpha wave started attenuating gradually, and became lower than that of the theta wave until 360 seconds. The average amplitude of the alpha wave until the first 270 seconds was app0roximately 8.0 , and it decreased to 0.91 by 360 seconds. The variation of the theta wave in the same period was changed from 2.22 to 3.0 and these results meant that the amplitude of the theta wave became higher than that of the alpha wave. As for the beta wave, the variation of its amplitude was less than 0.1. It is thought that the subjects were in the first-stage of sleep, because the theta wave was relatively higher than the alpha wave from 360 seconds onwards.

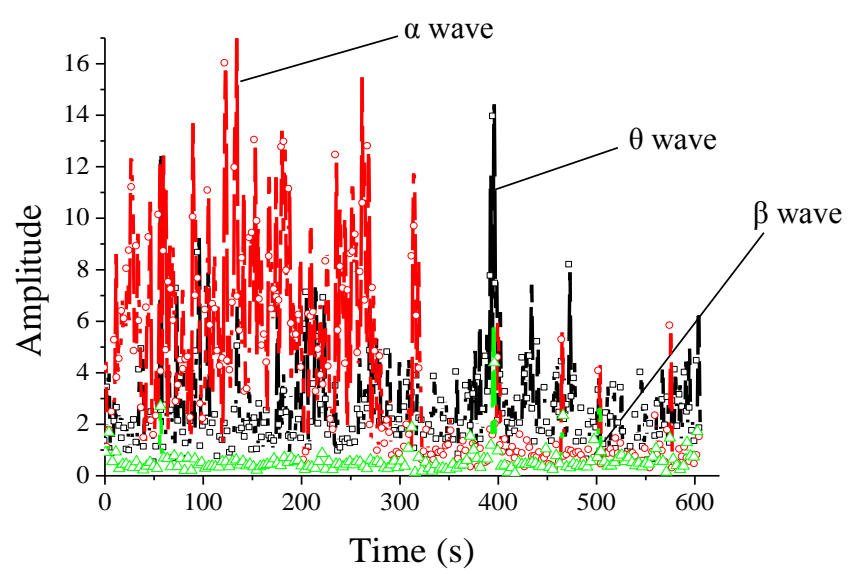

Fig.5 The amplitude of the each frequency element in the time domain

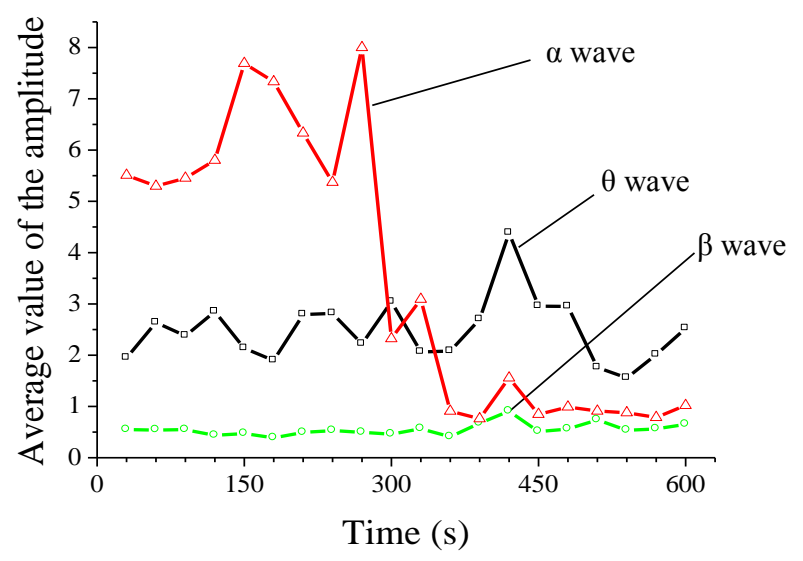

Fig.6 Average values of the amplitude of the alpha, beta and theta wave
To comprehend the relationship between the frequencies and the human state, we employed the IBRS in this study. As mentioned above, we thought that the subjects were in closed-eye wakefulness until 270 seconds as shown as in Fig.6. The average value of the IBRS in the same period in the Fig.7 was approximately 0.64. This represented that the amplitude of the alpha wave was higher than that of the beta wave and theta wave. Whereafter in the period from 270 to 360 seconds, the IBRS value sharply became higher than 1.5. It was thought that the amplitude of the alpha wave decreased compared to the other frequency elements, because the average value of the IBRS of period from 360 seconds to the end of the experiment was approximately 1.66. From the above result, it was found that to estimate the transition of human state from the rest state to sleep onsets during period from 270 seconds to 360 seconds by using the IBRS is possible.

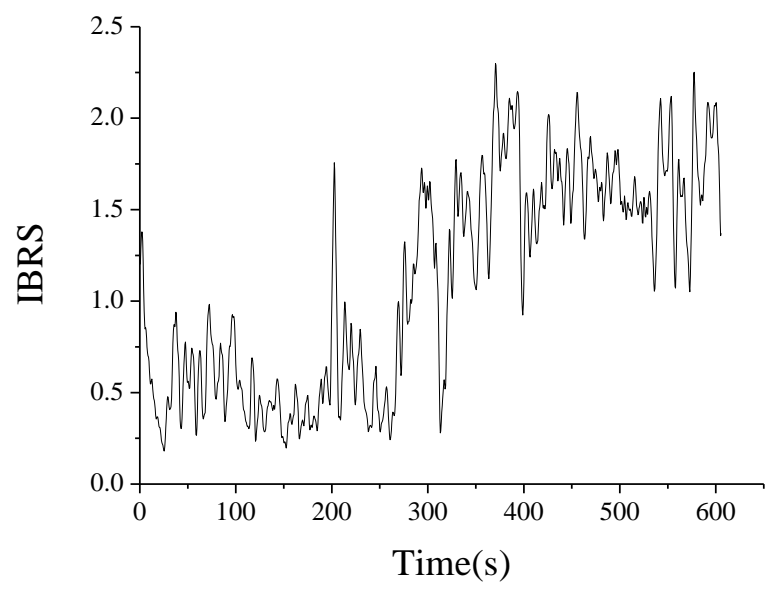

Fig.7 Transition of the IBRS in time domain

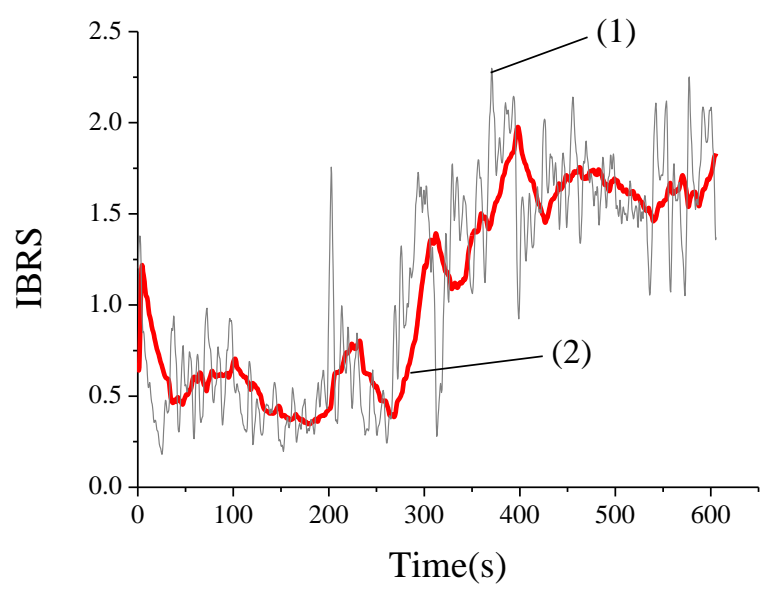

Fig. 8 The IBRS calculated from adjacent average
(1) 1 second
(2) 60 seconds 


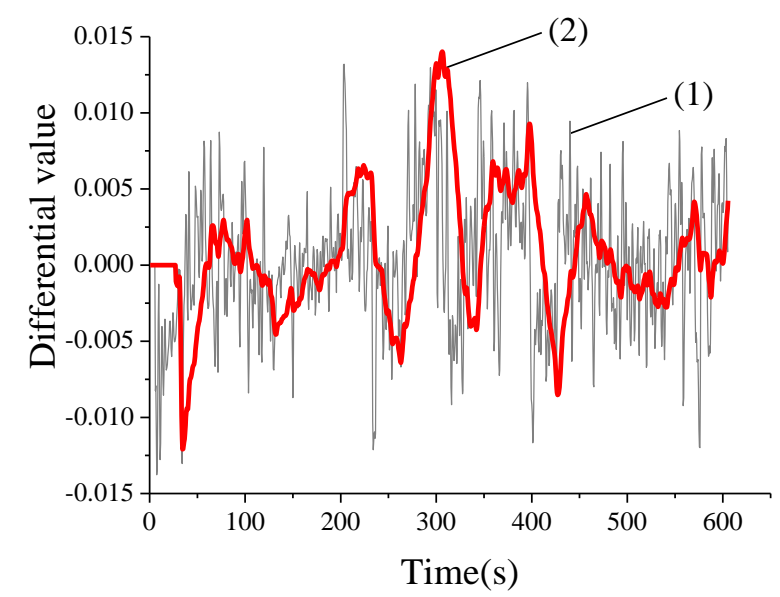

Fig.9 Differential value of the IBRS

(1) 1 second (2) 60 seconds
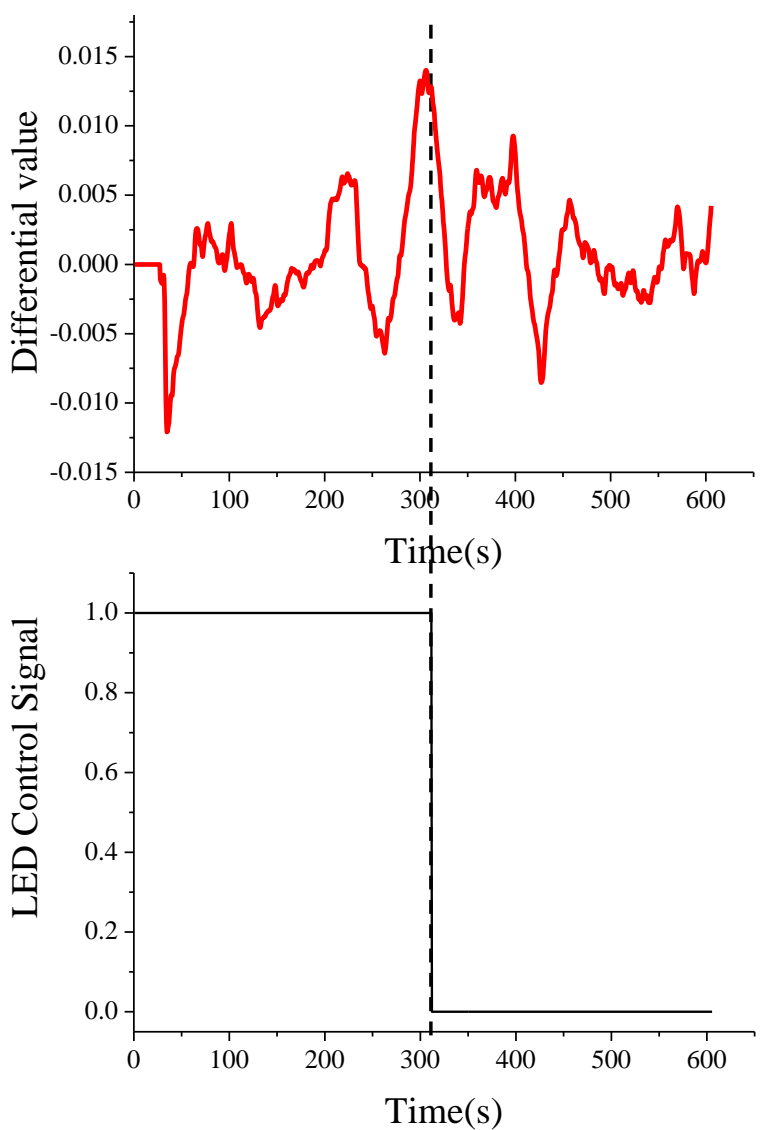

Fig.10 Control signal calculated from both the IBRS and differential value of the IBRS

Next, experiments using the fabricated Sleep Support System were carried out. The system turns on the LED when detecting wakefulness, and turns it off when detecting the sleep state. To understand the transition to the sleep state, observation from a broad perspective is important. To this end, the IBRS data was smoothed by calculating adjacent average over $60 \mathrm{~s}$, as the IBRS measured by the EEG fluctuates a lot from second to second (Fig. 8). The thin black line and the red line in the figure represent the IBRS per second, and the adjacent average over $60 \mathrm{~s}$, respectively. Calculating the adjacent average over 60 s eliminates sharp fluctuations, so we can evaluate the IBRS from a broad perspective. To aid the estimation of sleep onset, the change ratio of the IBRS was calculated every second using the smoothed data. Figure 9 shows the change ratio calculated at $1 \mathrm{~s}$ intervals and $60 \mathrm{~s}$ intervals. The sleep onset is clearly more easily detected using the smoothed change ratio calculated over the $60 \mathrm{~s}$ interval than over the $1 \mathrm{~s}$ interval. The transition from wakefulness to sleep onset is considered to occur within an approximately $60 \mathrm{~s}$ period between $250 \mathrm{~s}$ to $310 \mathrm{~s}$ after the beginning of the experiment. This means that unless the interval of the change ratio is set to capture that within $60 \mathrm{~s}$ period, there is a possibility that the transition could occur undetected. Therefore, the adjacent average over $60 \mathrm{~s}$ was used to determine the IBRS, and the change ratio was calculated at $60 \mathrm{~s}$ intervals using the smoothed IBRS. Figure 10 shows the generated signal for controlling the installed LED based on both the IBRS and change ratio of the IBRS. The high-level signal (1.0) and the low-level signal (0.0) indicate that the LED is on or off, respectively. The high-level signal was generated during the period from the experiment start to $250 \mathrm{~s}$ because the EEG data provided indicated an arousal-state based on the IBRS of 1.0 or less. The calculated change ratio shifted drastically between 250 $\mathrm{s}$ and approximately $310 \mathrm{~s}$, yielding a large value for the ratio. At the same time, the calculated IBRS also rose from a value of 0.5 or less to 1.4 or more. Therefore, this period was assumed to mark the transition from the arousal state to sleep onset. After approximately $310 \mathrm{~s}$, the sleep onset period was considered ended because the change ratio decreased under 80 percent while the IBRS remained in excess of 1.0. Thus, the low-level signal was generated, instructing the LED to turn off. From the above results, it is suggested that the proposed Sleep Support System, which estimates sleep-onset based on the IBRS calculated from EEG components and controls indoor conditions accordingly, is possible.

\section{Conclusions}

In this study, the estimation of sleep initiation using the brainwaves transmitted from the simple EEG sensor was researched for the proposed system. The IBRS calculated from the alpha wave, beta wave, and theta wave was employed to estimate the transition to the sleep-stage. The average value of the alpha wave and theta wave in the stage of closed-eye-wakefulness was, respectively, 5.3 and 2.9. In the next stage thought as the arousal-state, the amplitude of 
the theta wave was higher than that of the alpha wave. It was thought that estimation of the sleep-stage was possible by comparing each frequency elements. Next, the subject state was estimated using the IBRS. During the stage thought as the closed-eye wakefulness, the average value of the IBRS was 0.64 and was lower than one. In the next stage thought as the arousal stage, the average value of the IBRS was 1.66, which meant attenuation of the alpha wave. Next, the experiment controlling installed LED was carried out by using the fabricated Sleep Support System. The adjacent average of during 60 seconds was used to the IBRS, and the differentiate value was calculated at 60 seconds interval using the smoothed IBRS. The fabricated system seemed to perform well, because the LED was turned on during the arousal state and turned off during the sleep-onsets. From the above results, we found that the estimation of the transition from wakefulness to the sleep-stage was possible using the IBRS.

\section{References}

(1) Ministry of health, Labour and Welfare, http://www.seikatsusyukanbyo.com/statistics/disease/d yssomnia/ (accessed 2017-03-08).

(2) Y.Doi, "Prevalence and health impacts of sleep disorders in Japan", Journal of the National Institute of Public Health, Vol.61, No.1, pp.3-10, 2012.

(3) Makikawa, Yoshida, Nambu, Shiozaki, Okada, "Measurement technologies of mind and body condition in daily life for the development of human friendly products", CORONA publishers, 2010, in Japanese.
(4) M.Akita and Y.Midorikawa, "The examination of the detecting the sound signals in the human body for prediction of sleep in sleep-wake state", Institute of Electronics, Information, and Communication Engineers, Vol.108, pp.93-98, 2008.

(5) M.Akita, Y.Midorikawa, T.Kanechika and M.sakaguchi, "The measurement of the detecting the sound signals in the human body for prediction of sleep in sleep -wake state (The 2nd report), Institute of Electronics, Information, and Communication Engineers, Vol.109, pp.25-30, 2009

(6) J,Kiyotoki, T.Yamaguchi, M.Maeda and K.Inoue, " Extraction of detailed sleep information based on modified wavelet transform”, SICE, pp.1287-1289, Nov, 2011.

(7) NeuroSky, "MindSet Communications Protocol", NeuroSky application note (2011).

(8) S.Sanei and J.A.Chambers, "EEG Signal Processing", Wiley Interscience Publishing, 2007.

(9) M.Ikeno, M.Nakao, N.Katayama, and M.Yamamoto, "Dynamics of Polygraphic Signals during Sleep Stage Transition", Institute of Electronics, Information, and Communication Engineers, Vol.101, pp.31-38, 2001.

(10) On the subject of brainwave : digital medic Inc, http://www.digital-medic.com/waves (accessed 201703-08).

(11) T.Ichikawa, "An Invitation to Journey for Understanding of EEG”, Seiwa Shoten Publishers, 2006, in Japanese.

(12) K.Yoshii and K.Matsumoto, "Estimation of the Human State Based on the Index of Brain Resting State (IBRS) by Using Single-Channel Dry EEG Sensor”, ISTS 2015, Aug, 2015. 